\title{
Network Service Method under Cloud Environment of Wireless Sensor Networks in Disaster Situation
}

\author{
https://doi.org/10.3991/ijoe.v12i11.6231 \\ Chen Liang-wei \\ Chengdu Aeronautic Polytechnic, Sichuan, China
}

\begin{abstract}
The sensor networks are characterized by tiny, inexpensive, and advanced communication technology. However, wireless ad-hoc sensor networks (WASNs) have some issues such as the limited amount of energy, multipath fading, and some other issues when they applied in disaster situations. For reducing power consumption, we show mathematical analysis of energy saving, and enhanced error symbol probability. The simulation results prove our analysis. As for the multipath fading, we also show the mathematical analysis of fading channel characteristics and channel model, as well as how to mitigate the multipath fading by modulation and diversity techniques. From the simulation results, we prove the proposed system with amplify-and-forward (AF) relaying can achieve a $2.5 \mathrm{~dB}$ performance gain the BER $10^{-4}$ whereas the achievable improvement is almost a $4 \mathrm{~dB}$ at the BER $10^{-5}$ when using maximal ratio combining.
\end{abstract}

Index Terms-network service, cloud environment, disaster situation, wireless sensor networks

\section{INTRODUCTION}

Wireless sensor network usually consists of large number of sensor nodes with self-organized manner. And data gathering is one of the most fundamental and important operations in sensor networks. Sensor nodes sample local environment, and transmit the results to base station through the multi-hop routing, even to the remote user[1-2]. Currently, the power for sensor node is supplied by dry battery or fastener battery. So it is a challenging task to design energy-efficient data gathering protocols for wireless sensor networks. Moreover, low delay is a typical QoS requirement in wireless sensor networks, that's because low-delay data gathering can help to capture the abnormity in the monitoring area in time. Also, it is important to avoid the disaster and to decrease the loss of the disaster.

The proliferation of wireless computing and communication devices is leading to a huge in an information society. Communication between varieties of these devices can make it possible to offer unique and innovative type of services. In the past few years, a lot of wireless connectivity standards and technologies are appeared, which enable users to connect to a large group of computing and telecommunications devices by a simple way[3]. These types of technologies provide opportunities for speedy ad-hoc connections and the opportunity of automatic associations between devices. There are several such as technologies and standards and among the most important of these are Bluetooth, Home RF, and IEEE 802.11 standards.

An ad-hoc network is a group of wireless mobile terminals or nodes (or routers) that automatically forming a temporary infrastructure. The routers are free in lacking to usage of network their moving, and they can organize themselves arbitrarily; consequently, the network's wireless topology might quickly and randomly. Such a network can operate in a stand-alone manner, or can be connected to the Internet. Some features of this network such as mobility, mufti-hop routing, and large size merged with device heterogeneity, bandwidth and battery life time make the design of sufficient routing protocols a main challenge. In general, some form of the routing is essential in such situation, for the reason that two hosts might wish to exchange packets may not possibly be able to communicate directly[4].

A mobile user who desires to communicate in situation there is no network infrastructure available, such as firefighters require connection to an ambulance on the way to an emergency site. In such cases, a collection of mobile ad-hoc with wireless network interfaces could form a momentary network without the assistance of any established network infrastructure or centralized administration. The mobile ad-hoc network (MANET) group which is formed within Internet Engineering Task Force (IETF) is mainly focusing on to develop evolving M.ANET specifications and introduces them to the track of an Internet standard. Their goal is to support mobile adhoc networks. The goal is to support mobile ad-hoc networks by hundreds of ad-hoc routers and solve a challenge that facing this kind of network. However, a number of challenges that face ad-hoc networking are such as limited the wireless transmission range, the hidden node problems, and packet losses according to transmission errors, in addition to the mobility that induced route changes, and battery restrictions.

Mobile ad-hoc networks could improve the scope service area of access networks as well as providing wireless connectivity to the areas with poor signal or have no previously coverage. To enhance performance, the adhoc host should have the capability to adapt to the difference in performance and coverage besides to switch gateways when useful. Ad-hoc networking brings advantages like simple connection to access the networks, dynamic multichip wireless network structures, along with direct peer-to-peer communication. The property of multichip ad-hoc network requires a gateway to the wired backbone. It is necessary for this gateway to have a 
network interface in all sites of networks and must be a part of the two routing global and the wireless local adhoc routing. The users might benefit from ubiquitous networks in numerous ways. The user mobility allows users to switch between devices, move sessions while still getting the same personalized services.

\section{OVERVIEW}

A wireless ad-hoc network can be utilized to provide disaster management service applications, such as in crisis recovery, where the complete communication infrastructure is damaged and resorting, communication rapidly is very important. By using a wireless ad-hoc network, an infrastructure can be set up in hours instead of weeks, as is needed in the case of wired line communication.

There is another application example of a movable adhoc network is Bluetooth that is designed to support a personal area network (PAN) by removing the wires between different devices, for example, printers and personal digital assistants (PDAs). Worth mentioning that the well-known IEEE 802.11, Wireless Fidelity (WiFi) protocol is supporting wireless ad-hoc network scheme in the absence of a wireless access point.

Moreover, the idea of wireless ad-hoc network due to the U.S. Defense Advanced Research Projects Agency (DARPA) of developing packet radio network that was utilized in the 1970s. A wireless ad-hoc network is a group of mobile devices establishing a short live or wireless temporary network in the lack of a supporting structure. The mobile ad-hoc networks can be utilized in establishing efficient and dynamic communication that can be used for rescue, emergency operations, crisis relief efforts and military networking. The Bluetooth is using an idea of ad-hoc network. Bluetooth was first introduced in 1998, and it utilized radio waves to broadcast wireless data over short distances, and it can support many users in any environment. Thus, eight devices could communicate with each other in picante or a wireless Bluetooth form. At one time, ten of Bluetooth's devices can coexist in the same coverage area. These devices can work as both a client and a server. The connection has to be established to swap data between every two Bluetooth devices. For a connection establishment, a device must ask for a connection with the other device. The Bluetooth was based on the concept of advancing wireless interactions with a variety of electronic devices. These devices can be mobile phones, PDAs, and laptops with the appropriate chips could everyone communicate wirelessly with the other.

Yang's paper [5] studies the energy-delay-aware data gathering problems: coverage-preserving gathering gathering-oriented node placement and interference-free transmission scheduling. The author develops a fire monitoring system with sensor networks based on the correlative research results. The problem of coveragepreserving data gathering is to study the trade-off and optimization between the density of the active nodes and the transmission range between the nodes. In the dense network, it will produce the redundant data, thus wasting much energy. And the node's transmission range becomes longer in sparse network, which results in mass transmission cost.
Hence, Eason's [6] paper first defines the coveragepreserving data gathering problem, which mainly selects a certain number of nodes to construct an energy-efficient tree while preserving the original coverage. This problem can be formalized into integer linear programming, and Zhang [7] presents a heuristic algorithm, CPDG, to solve this problem. Simulation results show that CPDG can greatly improve the performance of the network. For example, it can conserve about $30 \%$ energy cost compared with famous PEDAP algorithm. To extend the network lifetime, an improved algorithm, BCPDG, mainly constructs the tree based on the remainder energy on each node when some nodes use their batteries up, thus can balance the energy cost among all nodes and extend the network lifetime.

In gathering-oriented placement problem, $\mathrm{m}$ data source nodes and base station are fixed in a given area, and how to efficiently place $n$ relaying nodes to minimize the total energy consumption. Zhang's [7] paper first designs an efficient placement algorithm for linear model, and presents a vector-based node placement algorithm for the planar network model. That is, a novel vector-based placement algorithm is used to calculate the positions of the relay nodes to reach an energy-efficient deployment for wireless sensor network which contains fixed source nodes and certain number of relay nodes. The experimental results illustrate that the network deployed by our algorithm saves $50 \%$ energy of that deployed by regular algorithm when the ratio of source- and relaynumber is $1: 2$. As in practical application systems, the numbers of nodes are often limited because of the cost, so our algorithm is of significant importance to construct low cost WSNs application system. Many applications, such as emergent succor and fire monitoring, etc., require that the nodes can report the data to the user in lower delay. Thus, it is necessary to study energy-delay-aware data gathering problem. It presents an energy-delay-efficient data gathering algorithm (SODG), which takes the transmission interference into considerations. This algorithm is fully distributed and localized, for it is only depending on one-hop neighbors' information. To minimize the delay, the algorithm permits the parallel transmission, thus it is difficult to avoid the interference among the nodes.

Zhang [8] first constructs Gabriel graph with local information, and build up data gathering tree on the Gabriel graph. It can conserve the energy when transmission on the Gabriel graph, because this avoids the longer transmission range. What's more, parallel transmissions can minimize the delay. The experimental results show that our algorithm is energy-delay-efficient. Especially, SODG algorithm can conserve 64\% energy compared with PEGASIS algorithm, and decrease $80 \%$ delay compared with ring- based gathering protocols. Based on the research on the data gathering problem and combined with the practical requirements, Zheng [9] develops a fire monitoring prototype system. His paper also introduces the system architecture, the functional modules of the system and key energy-conserve technologies on the different layers of the protocol stack. Now, this system is in the phase of debugging in the real environment. The contributions and novelties of his paper are as following: It is the first time that his paper presents coverage-preserving data gathering scheme, which studies the trade-off between the node density and transmission 
range. And the paper designs two energy-efficient data gathering algorithms, CPDG and BCPDG.

For node placement problem, Li's [10] paper designs a novel Vector-based algorithm to place the nodes efficiently. And the experiments show its efficiency. For real-time data gathering problem, Niu [11] designs a distributed and localized algorithm to schedule the nodes' transmissions, so as to achieve low delay and low energy cost. The paper explores the data gathering problem through computational geometry, which can be extended to solve other problems in the field of sensor network. More than the theoretical research, the paper describes a fire monitoring system based on the wireless sensor networks.

\section{EXPERIMENT RESULT}

Recently, there has been an important increase in utilize of wireless applications, that require higher bandwidth and dependability. New procedures such as diversity combining in wireless infrastructure have appeared to enhance wireless bandwidth and dependability. The combined signal can have a better SNR than that of the non-cooperative communications. Thus, cooperative communications are currently regarded as a hopeful approach to enhance spectral, power efficiency, and network coverage besides, to decrease the outage probability.

The relay selection is a vital tool for cooperative protocols. Appropriate relay selection systems can maximize transmission rate and dependability. Especially, relay selection in the cooperative communications can have important impacts on the network topology of mobile ad-hoc Networks (MANETs). Mobile ad-hoc network can be defined as a wireless communication in which terminals that not in direct transmission of each other need other terminals as a relay to forward data. As a result of the broadcasting nature of the media, this network normally adopts a cooperative transmission policy or another while data from a source to destination through multiple available routes. The essential idea is to forward data via the best selected or combined route to utilize the communication resources efficiently to achieve an overall performance with higher dependability; less transmits and/or higher broadcast rate. Channel fading in mobile ad-hoc networks is considered as a harmful phenomenon which can degrade the overall network performance.

Lately, multiple-input multiple-output technique has been introduced to enhance the dependability of the received signal and to support high data rates. Diversity technique also has been proposed to mitigate the result of channel. The concept of diversity is more prolonged to include cooperative networks, in which the notion of user cooperation diversity is applied. Moreover, the terminal in the cooperative networks at any time can operate as a sender, relay, or destination according to the network traffic and topology. Terminals close to the transmitter and/or receiver can operate as relay terminals. It forwards the source data to the desired receiver terminal via an independent relay channel, which is independent of the source and destination channel.

In figure 1, it shows each cluster of terminals that are positioned close together can swap information to make a virtual antenna array, that leading to a distributed multiple-input multiple-output (MINIO) system. That is to say, terminals on transmit side and indicated by dash line can exchange information to make a multiple-antenna transmitter. Furthermore, terminals on the receiver side and indicated by dash line can exchange information to shape a multiple-antenna receiver and same can happen in the relay side. Since each terminal has a dissimilar channel to each receiver, this cooperative ad-hoc MIMO system has performance benefits in terms of multiplexing and diversity. Moreover, the multiple antennas can be utilized on transmit or receiver side to guide the beam in the direction of the planned receiver, by this means reducing interference and multi-path fading.

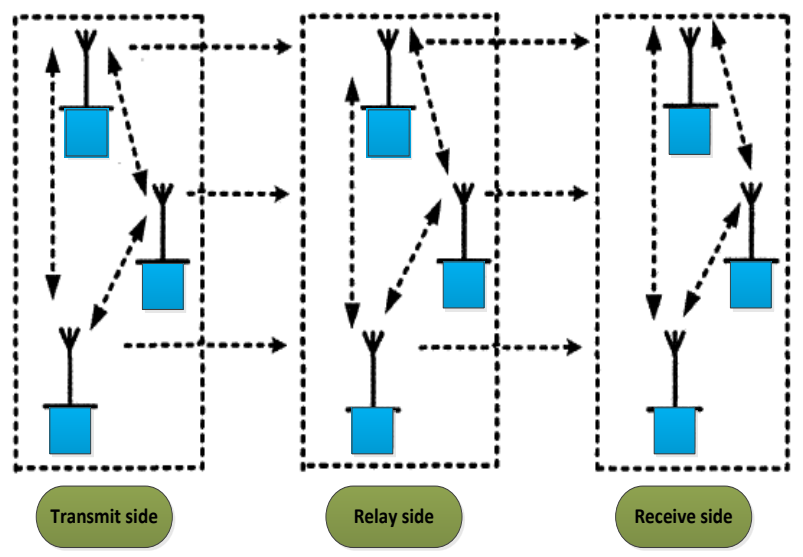

Figure 1. The basic model

The basic equation of key algorithm is shown as the equation (1):

$$
(N, s k) \leftarrow \operatorname{Key}\left(1^{k}\right)(1)
$$

This formula is used to generate file checksum parameter which is denoted by:

$$
\begin{aligned}
& r \leftarrow\{0,1\}^{k} ; s k \leftarrow\{e, d, r\} ; \\
& \text { Output }\{N, s k\}
\end{aligned}
$$

The Euler function is:

$$
\phi(N)=(p-1)(q-1)
$$

Then choose an integer $\mathrm{e}$ to satisfy the following equation 4:

$$
\left\{\begin{array}{l}
1<e<\phi(N) \\
\operatorname{gcd}(e, \phi(N))=1
\end{array}\right.
$$

Then finally export $(\mathrm{N}, \mathrm{sk})$ in Tag algorithm, we can get the optimization equation (5):

$$
\left(T_{0}, T_{2}, \ldots T_{n-1}\right) \leftarrow \operatorname{Tag}(p k, s k, m)(5)
$$

The formula generates labels for each file block.

$$
\begin{aligned}
& \text { for }(j=0 ; j \leq n-1 ; j++) ;(6) \\
& \left\{W_{j}=r^{*}(j+1) ; T_{i}\right. \\
& \left.=\left[h\left(W_{j}\right) * m_{j}\right]^{c} \bmod N\right\} ; \\
& \quad \text { Output }\left(T_{0}, T_{2}, \ldots T_{n-1}\right) ;(8)
\end{aligned}
$$

And local fractional integral of $f(x)$ defined by Eq.9. 


$$
\begin{aligned}
& { }_{a} I_{b}^{(\alpha)} f(t)=\frac{1}{\Gamma(1+\alpha)} \int_{a}^{b} f(t)(d t)^{\alpha} \\
& =\frac{1}{\Gamma(1+\alpha)} \lim _{\Delta t \rightarrow 0} \sum_{j=0}^{j=N-1} f\left(t_{\mathrm{j}}\right)\left(\Delta t_{\mathrm{j}}\right)^{\alpha}
\end{aligned}
$$

Its local fractional Hilbert transform, denoted by $f_{x}^{H, \alpha}(x)$ is defined by

$$
\begin{aligned}
& H_{\alpha}\{f(t)\}=\hat{f}_{H}^{\alpha}(x) \\
& =\frac{1}{\Gamma(1+\alpha)} \int_{R} \frac{f(t)}{(t-x)^{\alpha}}(d t)^{\alpha}
\end{aligned}
$$

Where $\mathrm{x}$ is real and the integral is treated as a Canchy principal value, that is,

$$
\begin{aligned}
& \frac{1}{\Gamma(1+\alpha)} \int_{R} \frac{f(t)}{(t-x)^{\alpha}}(d t)^{\alpha} \\
& =\lim _{\varepsilon \rightarrow 0}\left[\frac{1}{\Gamma(1+\alpha)} \int_{-\infty}^{x-\varepsilon} \frac{f(t)}{(t-x)^{\alpha}}(d t)^{\alpha}+(11)\right. \\
& \left.\frac{1}{\Gamma(1+\alpha)} \int_{x+\varepsilon}^{\infty} \frac{f(t)}{(t-x)^{\alpha}}(d t)^{\alpha}\right]
\end{aligned}
$$

To obtain the inverse local fractional Hilbert transform, write again Eq. (11) as

$$
\begin{aligned}
& \hat{f}_{H}^{\alpha}(x)=\frac{1}{\Gamma(1+\alpha)} \int_{-\infty}^{\infty} \frac{f(t)}{(t-x)^{\alpha}}(d t)^{\alpha} \\
& =\frac{1}{\Gamma(1+\alpha)} \int_{-\infty}^{\infty} f(t) g(x-t)(d t)^{\alpha} \\
& =f(x) * g(x),
\end{aligned}
$$

The equation of motion is as follows:

$$
\partial_{j}\left(C_{i j k l} \partial_{k} u_{l}+e_{k i j} \partial_{k} \varphi\right)-\rho \ddot{u}_{i}=0
$$

Under the linear theory, that is:

$$
\partial_{j}\left(e_{i j k l} \partial_{k} u_{l}-\eta_{k i j} \partial_{k} \varphi\right)=0
$$

The figure 2 shows the basic framework of cloud model with the figure 3 shows the cloud architecture in network model. One of the most excellent systems to alleviate the effects of multipath fading is diversity combining of separately fading signal paths. The diversity combining exploits the reality that independent signal paths experiencing deep fades at the same time. These small possibilities of paths are merged in such a way to decrease the fading of the resultant signal.

The aim in implementing the diversity system is to use extra self-governing signal pathways to the received signal-to-noise ratio (SNR). Diversity can give scheme performance at comparatively low cost; it is not similar equalization; diversity needs no training overhead. Here, the error performance improvement can be achieved by utilizing the diversity techniques.

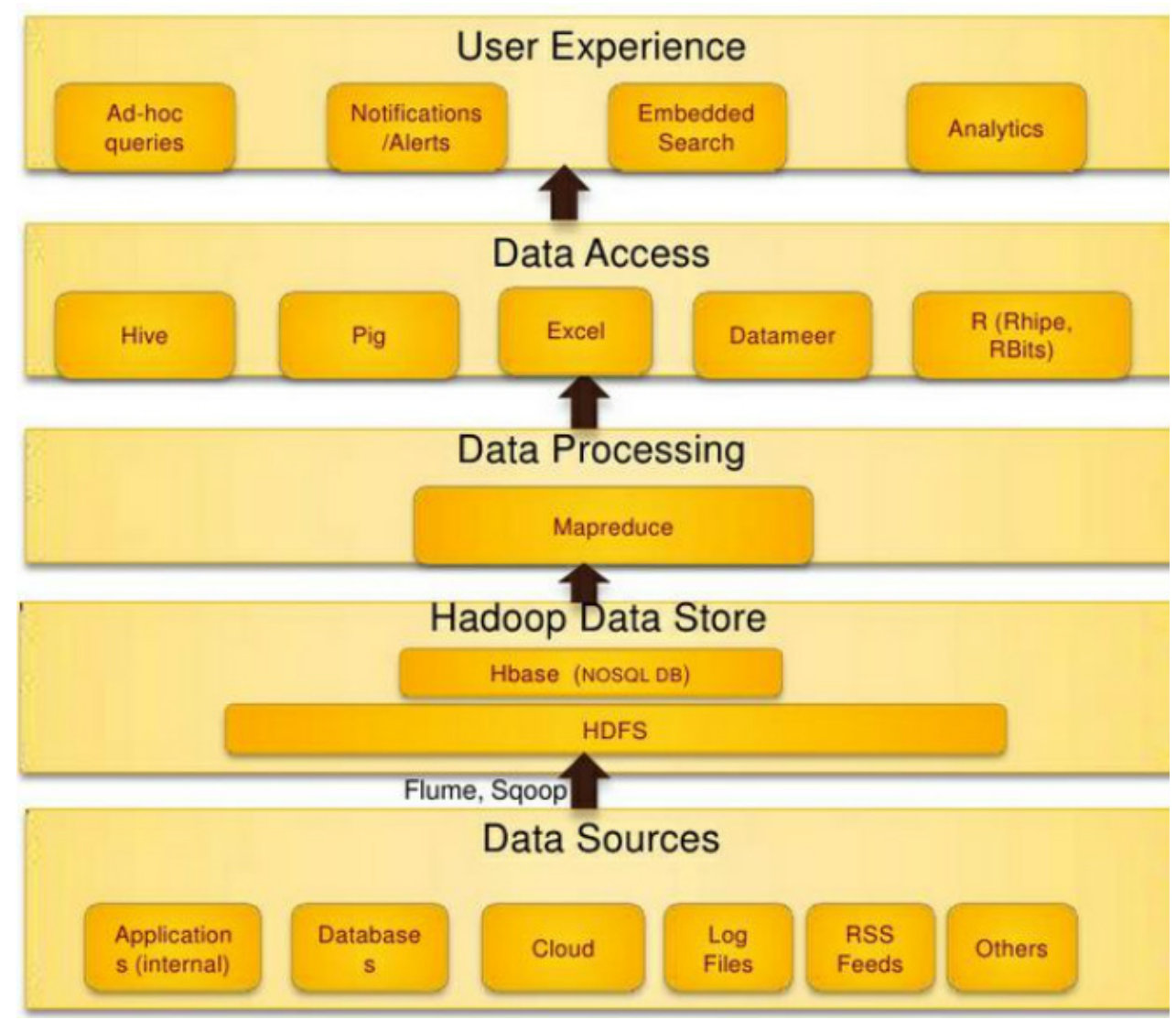

Figure 2. The basic framework of cloud model 


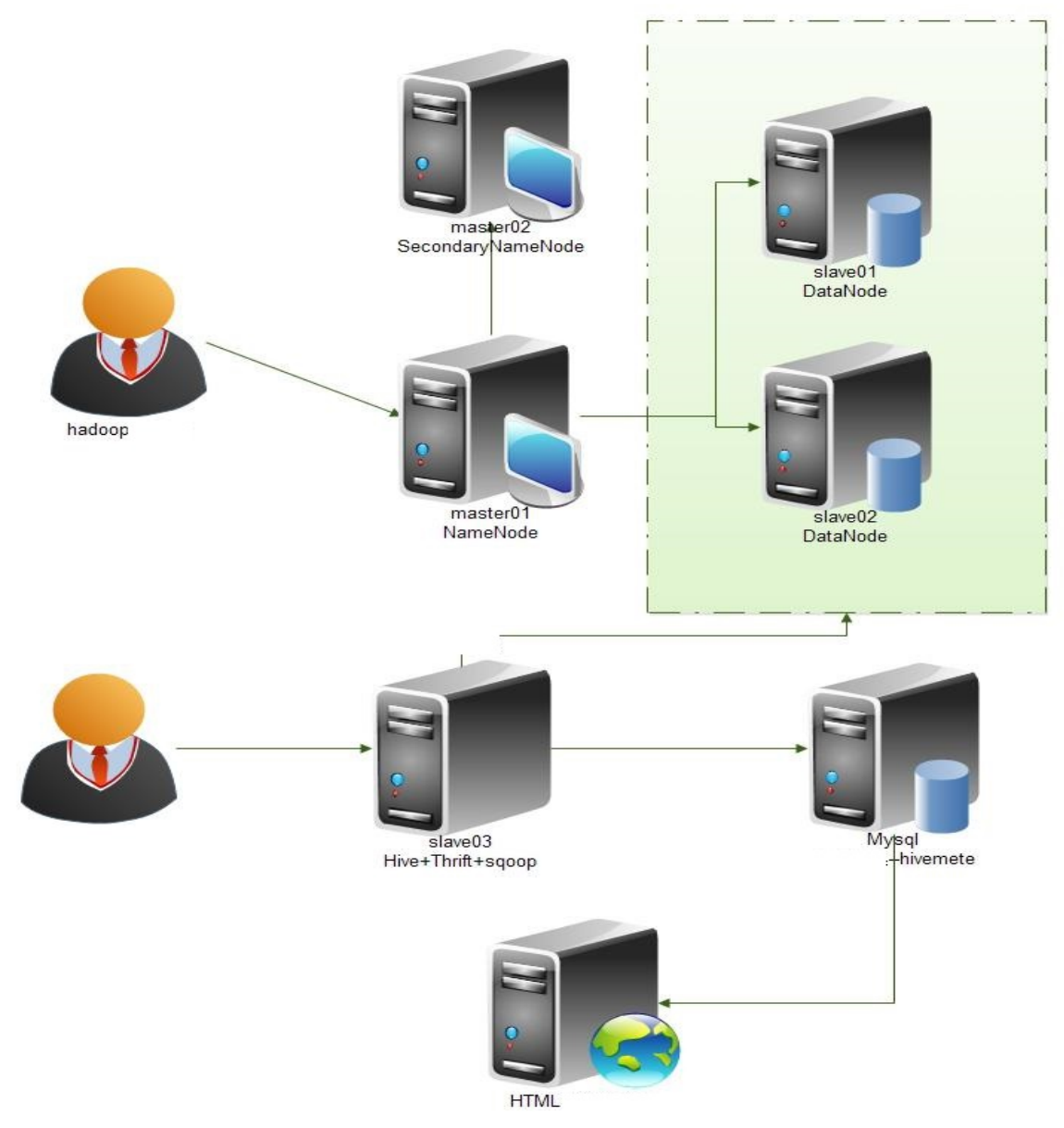

Figure 3. The cloud architecture in network model

\section{DISCUSSION}

The performances of the modulation techniques over the fading channel are shown in figures below. The bit error probability of PSK over fading channels is obtained in figure 4. Figure 5 illustrates the performance of QAM over multipath fading channel. The comparison of bit error probability between PSK and QAM over fading channels is shown in the figures, from which it observed that the performance of QAM is better than of the PSK. Then the result is extended to include the performance of modulation techniques of PSK and QAM over Rician fading channel with the diversity is also shown in this figures.

The computers' processing power and massive data storage capacity have improved a lot by now, and some general patterns can be discovered from all kinds of practical datasets which are accumulated in the objective world. To find out the laws, statistics, data mining, machine learning and some other related technologies are used. In the past decade, researchers have found that the network structure is widely included in nature and human society, and reveal some unique structural features in real world gradually. With the development of network science, the network-based analysis and graph mining have been paid more and more attentions and are widely used in the physical, biological, political, economic, Internet, engineering development and social life and so on. Researchers process the datasets into network structure, and use graph theory, data mining to reveal the useful patterns.

By comparing the results of stage I, and stage II, that in equation (8) and (9), one can see that more power is allocated to the source at what time diversity combining is in use at the destination. This because diversity combining is used, the signal transmitted by the source shall be used for detection at both the relay and the destination and is more essential to accomplishing a higher performance in the cooperative transmission. The amplify-and-forward (AF) relaying can achieve a $2.5 \mathrm{~dB}$ performance gain the BER $10^{-4}$ whereas the achievable improvement is almost a $4 \mathrm{~dB}$ at the BER $10^{-5}$ when using maximal ratio combining.

\section{CONCLUSION}

Sensor nodes sample local environment, and transmit the results to base station through the multi-hop routing, even to the remote user. Currently, the power for sensor node is supplied by dry battery or fastener battery. So it is a challenging task to design energy-efficient data gathering protocols for wireless sensor networks. Moreover, low delay is a typical QoS requirement in wireless sensor networks, that's because low-delay data gathering can help to capture the abnormity in the monitoring area in time. The experimental results illustrate that the network deployed by our algorithm saves $50 \%$ energy of that deployed by regular algorithm when the 
ratio of source- and relay-number is $1: 2$. As in practical application systems, the numbers of nodes are often limited because of the cost, so our algorithm is of significant importance to construct low cost WSNs application system. Many applications, such as emergent succor and fire monitoring, etc., require that the nodes can report the data to the user in lower delay.

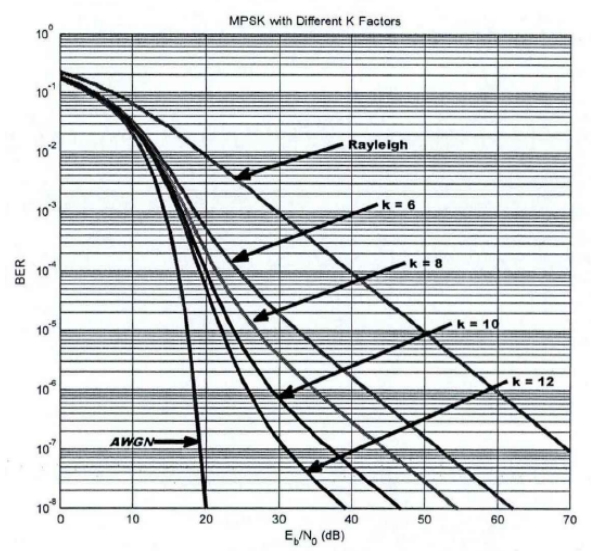

Figure 4. PSK experiment over fading channel

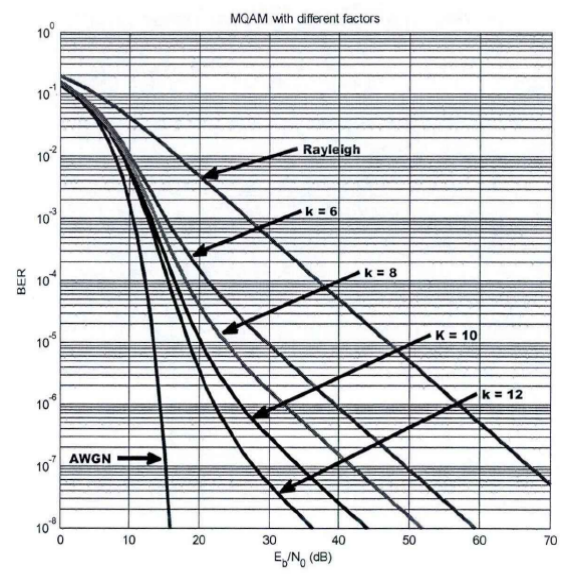

Figure 5. QAM experiment over fading channel

However, wireless ad-hoc sensor networks (WASNs) have some issues such as the limited amount of energy, multipath fading, and some other issues when they applied in disaster situations. For reducing power consumption, we show mathematical analysis of energy saving, and enhanced error symbol probability. The simulation results prove our analysis. Mobile ad-hoc network can be defined as a wireless communication in which terminals that not in direct transmission of each other need other terminals as a relay to forward data. As a result of the broadcasting nature of the media, this network normally adopts a cooperative transmission policy or another while data from a source to destination through multiple available routes. The essential idea is to forward data via the best selected or combined route to utilize the communication resources efficiently to achieve an overall performance with higher dependability; less transmits and/or higher broadcast rate. As for the multipath fading, we also show the mathematical analysis of fading channel characteristics and channel model, as well as how to mitigate the multipath fading by modulation and diversity techniques. From the simulation results, it is proved that amplify-and-forward (AF) relaying can achieve a $2.5 \mathrm{~dB}$ performance gain the BER $10^{-4}$ whereas the achievable improvement is almost a $4 \mathrm{~dB}$ at the BER $10^{-5}$ when using maximal ratio combining.

\section{REFERENCES}

[1] H. Jing, "Node deployment algorithm based on perception model of wireless sensor network," International Journal of Automation Technology,vol.9, no.3, pp. 210-215, April 2015. https://doi.org/10.20965/ijat.2015.p0210

[2] H. Jing, "Routing optimization algorithm based on nodes density and energy consumption of wireless sensor network," Journal of Computational Information Systems, vol. 11, no.14, pp. 50475054, July 2015.

[3] T. Yang, B. Yu, H. Wang, et al., "Cryptanalysis and improvement of Panda - public auditing for shared data in cloud and internet of things," Multimedia Tools and Applications, pp. 1-18, December 2015. https://doi.org/10.1007/s11042-015-3139-7

[4] H. Yang, et al., "Toward resilient security in wireless sensor networks," Proceedings of the 6th ACM international symposium on Mobile ad hoc networking and computing ACM, pp.34-45, 2015.

[5] G. Eason, B. Noble, and I. N. Sneddon, "On certain integrals of Lipschitz-Hankel type involving products of Bessel functions," Phil. Trans. Roy. Soc. London, vol. A247, pp. 529-551, April 1955. https://doi.org/10.1098/rsta.1955.0005

[6] D. Zhang, et al., "An Energy-Balanced Routing Method Based on Forward-Aware Factor for Wireless Sensor Networks," IEEE Transactions on Industrial Informatics, vol. 10, no. 1, pp. 766-773, 2014. https://doi.org/10.1109/TII.2013.2250910

[7] D. Zhang, et al., "An Energy-Balanced Routing Method Based on Forward-Aware Factor for Wireless Sensor Networks," IEEE Transactions on Industrial Informatics, vol. 10, no. 1, pp. 766-773, 2014. https://doi.org/10.1109/TII.2013.2250910

[8] X. Zhang, et al., "Rotation-based privacy-preserving data aggregation in wireless sensor networks," ICC 2014 - 2014 IEEE International Conference on Communications, pp. 4184-4189, 2014. https://doi.org/10.1109/icc.2014.6883977

[9] J. Zheng, et al., "Auction-based adaptive sensor activation algorithm for target tracking in wireless sensor networks," Future Generation Computer Systems, vol. 39, no. 1, pp.88-99, 2014. https://doi.org/10.1016/j.future.2013.12.014

[10] Z. Li, et al. "A low latency, energy efficient MAC protocol for wireless sensor networks," International Journal of Distributed Sensor Networks, vol. 10, no. 6, pp.1-9, 2015. https://doi.org/10.1155/2015/946587

[11] J. Niu, et al., "R3E: Reliable Reactive Routing Enhancement for Wireless Sensor Networks," IEEE Transactions on Industrial Informatics, vol. $10, \quad$ no. $1, \quad$ pp.784-794, 2014. https://doi.org/10.1109/TII.2013.2261082

\section{AUTHOR}

Chen Liang-wie is with the Department of information and Engineering, Chengdu Aeronautic Polytechnic, Sichuan, China (35839769@qq.com).

The paper is supported by Sichuan Province Natural Science Key Project: Application Research on Informationization Construction of the cloud computing, leading reseacher of the project( No. 15ZA0399). Submitted 09 September 2016. Published as resubmitted 12 November 2016. 